\title{
Digital Analysis of Nasal Airflow Facilitating Decision Support in Rhinosurgery
}

\author{
Thomas Hildebrandt, MD ${ }^{1}$ Jan Joris Brüning, $\mathrm{MSc}^{2}$ Hans Lamecker, $\mathrm{PhD}^{3}$ Stefan Zachow, PhD \\ Werner J. Heppt, MD ${ }^{5}$ Nora Schmidt, MD ${ }^{6}$ Leonid Goubergrits, PhD $^{2}$
}

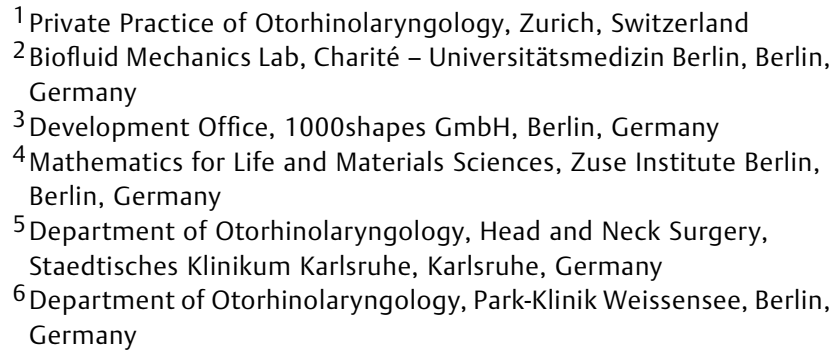

Address for correspondence Thomas Hildebrandt, MD, Private Practice of Otorhinolaryngology, Zurich 8005, Switzerland (e-mail: th@dr-hildebrandt.ch).

Facial Plast Surg 2019;35:3-8.

\begin{abstract}
Keywords

- nasal airflow simulation

- nasal breathing

- statistical shape model

- diANA

- nasal obstruction

- rhinorespiratory homeostasis

Successful functional surgery on the nasal framework requires reliable and comprehensive diagnosis. In this regard, the authors introduce a new methodology: Digital Analysis of Nasal Airflow (diANA). It is based on computational fluid dynamics, a statistical shape model of the healthy nasal cavity and rhinologic expertise. diANA necessitates an anonymized tomographic dataset of the paranasal sinuses including the complete nasal cavity and, when available, clinical information. The principle of diANA is to compare the morphology and the respective airflow of an individual nose with those of a reference. This enables morphometric aberrations and consecutive flow field anomalies to localize and quantify within a patient's nasal cavity. Finally, an elaborated expert opinion with instructive visualizations is provided. Using diANA might support surgeons in decision-making, avoiding unnecessary surgery, gaining more precision, and target-orientation for indicated operations.
\end{abstract}

Impaired nasal breathing is a frequent problem doctors have to deal with. Estimations suggest that up to $30 \%$ of the population in Europe and North America suffer from a recurrent or permanent nasal airway obstruction. ${ }^{1,2}$

There are three fundamental etiologic groups, and they can be interrelated or coexisting:

Group 1: Deformation of the nasal framework with consecutive changes of the flow domain of the nasal cavity. The resulting flow field anomalies can also alter the mucous membranes or erectile tissue with retroaction on the flow domain. $^{3}$

Group 2: Chronic inflammatory diseases or substancerelated reactions of the mucous membranes.

Group 3: Disturbed reception and/or perception of the nasal airflow.
In groups 2 and 3 , the first option of treatment is to eliminate the primary causes or rather the underlying disease. In contrast, group 1 patients can be helped in the first instance by surgical means restoring the nasal framework and the nasal cavity's flow domain. For this, objective information about the nasal airflow is essential. Currently, rhinomanometric methods developed by Vogt and Mlynski are considered the benchmark in this regard., ${ }^{4,5}$ In fact, they are only able to provide the value of the total nasal resistance of each side, which is sufficient for documentation of clear findings. However, these methods are lacking in details of the airflow within the nasal cavity. Hence, they are not eligible for identification of a flow-compromising site or structure. Furthermore, the value of the total nasal resistance is not one-to-one attributed to only a particular morphology of the
Issue Theme New Tools and Proven Techniques in Rhinoplasty; Guest Editors: Enrico Robotti, MD, and Werner J. Heppt, MD
Copyright (C) 2019 by Thieme Medical Publishers, Inc., 333 Seventh Avenue, New York, NY 10001, USA. Tel: +1(212) 584-4662.
DOI https://doi.org/ 10.1055/s-0039-1677720. ISSN 0736-6825. 
nasal cavity. This might be a reason for arbitrariness and polypragmasia in functional rhinosurgery. It should also be pointed out that a low nasal resistance does not automatically ensure a physiologic nasal airflow and inner milieu of the nasal cavity. Not least, the relatively high rate (approximately one fourth or $27 \%$ ) of unsatisfactory results of functional surgery on the nasal framework indicates the need to improve the preoperative diagnostic measures. ${ }^{5,6}$

In terms of this necessity, we introduce our methodology of comprehensive assessment of nasal breathing. It is based on airflow simulation (computational fluid dynamics [CFD]) in conjunction with a statistical shape model (SSM) of the healthy nose and an enhanced physiologic approach to nasal breathing-the concept of rhinorespiratory homeostasis (CRRH). ${ }^{7}$

\section{Fundamentals and Prerequisites}

Digital Analysis of Nasal Airflow (diANA) merges three innovative methods or scientific approaches described briefly below. It needs the skills of engineers (fluid mechanics), computer scientists (image processing), and medical doctors (otorhinolaryngology) to implement and execute this service.

\section{Computational Fluid Dynamics}

Computational fluid dynamics is an advanced technology that is established in the industry as well as increasingly used in medicine. In particular, it allows for simulation of the fluid flow within complex geometries by numerically solving equations describing conservation of energy, mass, and momentum. CFD requires prior reconstruction of the flow domain and its discretization with finite volumes, generating a grid. Then, calculation in consideration of the boundary conditions and subsequent visualization can be performed. ${ }^{8-10}$

Typical medical applications are exploration of the blood flow in the heart, aorta, or brain vessels. ${ }^{11,12}$ Investigations of the intranasal airflow using CFD is the subject of research for many years. ${ }^{13-22}$ In contrast to rhinomanometry, CFD is able to provide user-defined parameters of the intranasal airstream in a high spatial and temporal resolution. Required is a Digital Imaging and Communications of Medicine (DICOM) dataset derived from a computed tomography (CT scan) or optionally from a cone beam $\mathrm{CT}$ of the paranasal sinuses. Its resolution should be according to the recommendations for navigated surgery, and the nasal cavity has to be completely captured from the tip up to the choanae. Then, sufficient reconstruction of the nose's three-dimensional (3D) geometry is possible after segmentation. Our specific CFD workflow including the used modeling and boundary conditions is in accordance with those of a prior study. ${ }^{13}$

\section{Statistical Shape Model of the Nasal Cavity}

The geometry of the nasal cavity exhibits an enormous heterogeneity, which causes corresponding variety of the respective airstream. ${ }^{23}$ Besides that, there are naturally occurring characteristic morphological structures such as the septum, isthmus nasi, and turbinates. The latter form the three nasal meatus on the lateral nasal wall. These topolo- gical conditions complicate the comparison of individual noses and, hence, the related airstream. It makes it challenging to distinguish between physiologic and pathologic airflow. We address this problem using a SSM of the nasal cavity. The model was generated from a population of 25 healthy noses (50 nasal cavities, right and left side) of subjects who did not report impaired nasal breathing. The patients were selected by one and the same examiner (ear, nose, and throat [ENT] specialist). The examiner also excluded the existence of inconsistent findings. The individual geometries were reconstructed from segmented CT scans of the paranasal sinuses available through the routine ENT practice run by one of the authors. Subsequently, a specific mathematical algorithm, described by Lamecker and colleagues in 2009 and 2016, was applied to obtain an appropriate SSM of the healthy nasal cavity. ${ }^{24,25}$ The respective averaged geometry shows all regular anatomical landmarks. In addition, the calculated airflow exhibits all characteristic features, which are described in the literature as well as observed in our own simulations. ${ }^{14,15,17,19-21,23}$ We presume that the mean geometry of the SSM represents normal morphology of the nasal cavity determining physiologic nasal airflow. Consequently, it can serve as the reference used in diANA intending to delineate impaired from nonimpaired nasal breathing and to reveal the related noticeable findings in case of problems (-Figs. 1 and 2 ).

\section{Concept of Rhinorespiratory Homeostasis}

Concept of rhinorespiratory homeostasis is an alternative approach to nasal breathing. It focuses on the parietal effect of the inspiratory and expiratory intranasal airstream referring to wall shear stress, whereas the conventional view mainly considers the inhaled air., ${ }^{3,6}$ The wall shear stress parameter describes the amount of shear force on the nasal wall through the flowing air. It depends on the near wall flow velocity and correlates with the mass and heat transfer occurring between the wall and air. Several biological and physical reactions in/on epithelial and endothelial tissues are attributed to wall shear stress. ${ }^{26-30}$ In this context, CRHH addresses the link of the epithelium lining liquid fluid and nasal breathing. ${ }^{3}$ One implication drawn is that the existence of a normal intranasal flow field in conjunction with adequate secretion is necessary as well as sufficient for maintaining a physiologic inner milieu (prerequisite for nasal functions and airstream perception), provided there are no adverse factors such as inflammatory or toxic influences and destructed epithelium. It suggests that consideration of temperature and humidity might be dispensable contemplating the link between the interaction of the flow field and inner milieu of the nose.

\section{Methodology}

diANA starts with patient selection and acquisition of detailed information about medical history, clinical findings, and rhinomanometry, when available. It can be provided either through personal examination, via teleconsultation, or exchange with the attending doctor. 

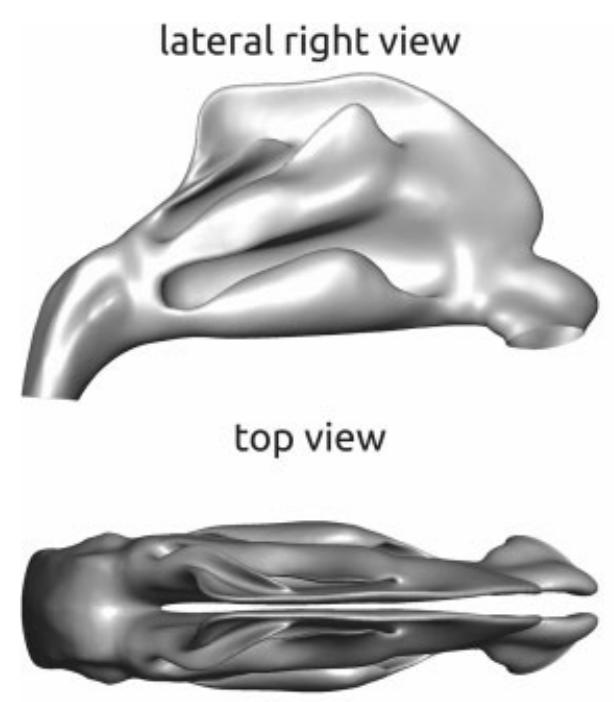

frontal view

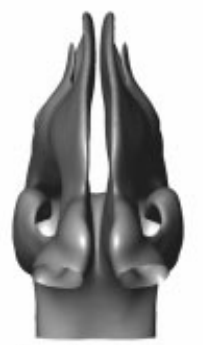

back view

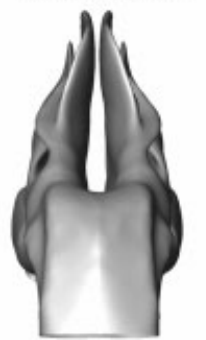

lateral left view

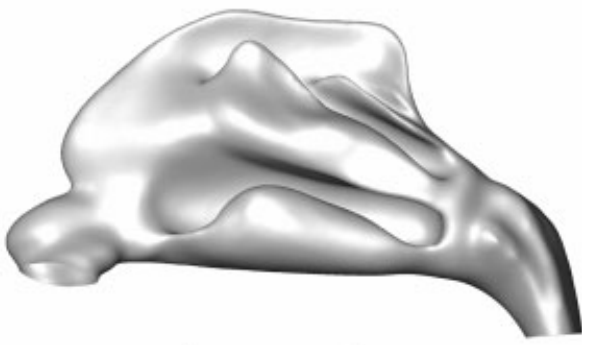

bottom view

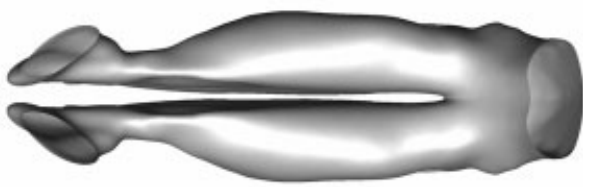

Fig. 1 Mean geometry of the statistical shape model derived from healthy nasal cavities viewed from diverse directions, exhibiting the entirety of the common anatomical landmarks.
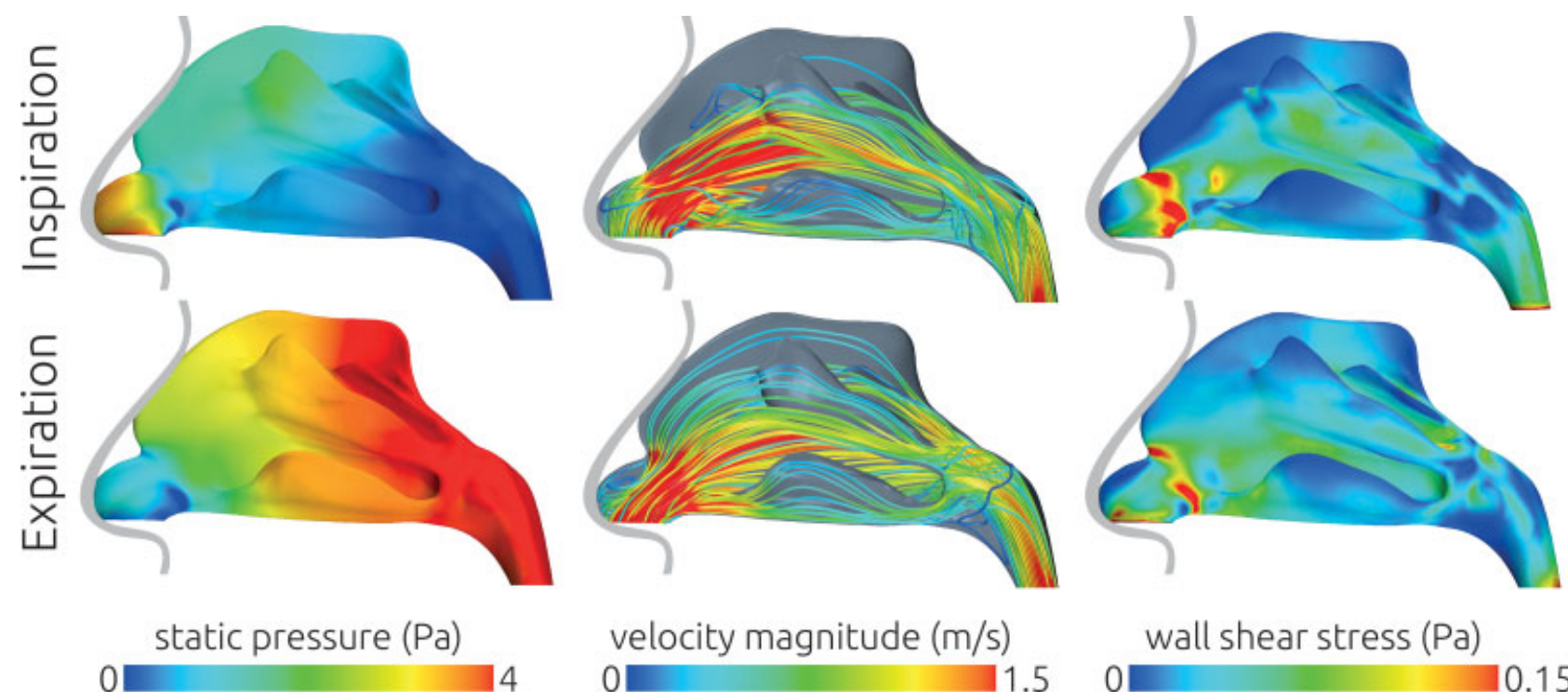

velocity magnitude $(\mathrm{m} / \mathrm{s})$
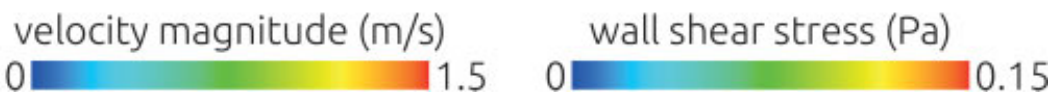

Fig. 2 Calculated steady airflow $(12 \mathrm{~L} / \mathrm{min}$ according to $6 \mathrm{~L} / \mathrm{min}$ respiratory minute volume at rest) in the mean geometry of the statistical shape model derived from healthy nasal cavities for inspiration and expiration, displayed from left to the right side: pressure field, velocity field, and distribution of wall shear stress in the left nasal cavity.

The DICOM data set of the CT scan has to be anonymized prior to delivery for segmentation and subsequent reconstruction of the 3D geometry, which the workflow of CFD initiates.

The calculation of the following flow field parameters is sufficient for diANA: flow velocity, wall shear stress, pressure field, pressure drop, and the total nasal resistance of each nasal cavity. We do not take into account temperature and humidity.

The nose is regarded as parallel-connected conduits. Generally, we consider laminar steady airflow of $12 \mathrm{~L} / \mathrm{min}$ according to breathing at absolute rest with a respiratory minute volume of $6 \mathrm{~L} / \mathrm{min}$.

The obtained patient-specific nasal geometry and the related flow field parameter values are compared with those of the SSM or the derived mean geometry, to identify the differences (-Fig. $\mathbf{3}$ ).

The juxtaposition can be executed on three levels. Usually, visual comparison of the airstream patterns, including the calculated total resistances as well as the nasal cavity's morphologies with consideration of clinical information, is sufficient to make a valuable judgment. This complies exclusively with qualitative assessment, which is level one. In certain cases, we map the patient's geometry and flow field onto the mean geometry of the SSM and its flow field, respectively. This allows for semiquantitative calculation of deviations in a point-to-point mode, which is level two. However, there are no predictions possible whether or not 


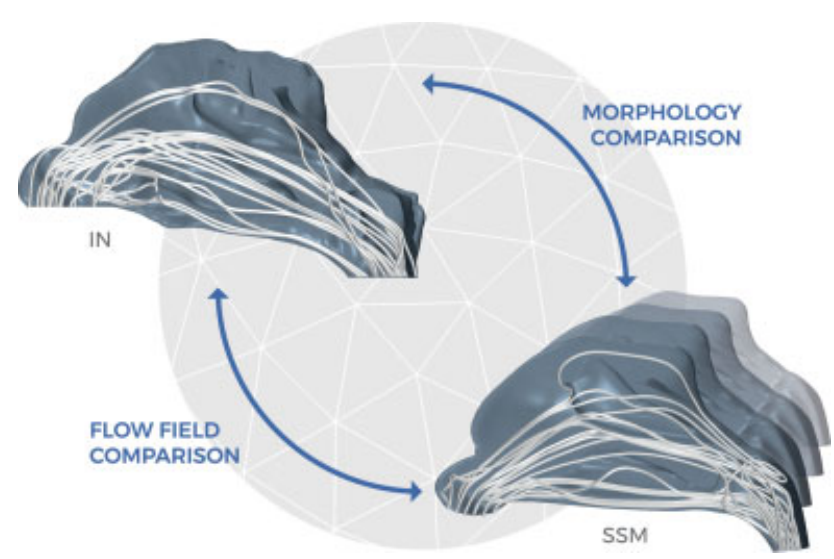

Fig. 3 IN: Individual nasal cavity with respective airflow. SSM: Statistical shape model of the nasal cavity/derived mean geometry with respective airflow.

the detected differences are statistically significant. Only mathematical transformation of the patient's data to those of the SSM can gain fully quantitative information about aberrations, which is level three.

Finally, after compiling all information and data, elaboration of an expert opinion including instructive visualizations is executed, using the synergy of advanced technology and rhinologic expertise. This might support the attending doctor's decisions.

\section{Discussion}

\section{Added Value}

In contrast to rhinomanometry, the nose is no longer considered a black box but as a system of two parallel conduits. In addition, to our knowledge, diANA is the first and currently only tool that employs a SSM of the healthy nose as a reference for the evaluation of nasal breathing. This enables morphometric aberrations and consecutive flow field anomalies to localize and quantify within the nasal cavity. Furthermore, the wall shear stress parameter provides a measure of the mass and heat transfer as well as mechanical forces. Both values stimulate receptors in the epithelium that is required for the perception of the nasal airstream and regulating processes.

The isthmus nasi is the essential bulk flow formation structure. ${ }^{31-34}$ However, it is impossible to gain all flowrelevant parameters of this structure through a clinical or even CT scan examination. Only 3D reconstruction of the nasal cavity's geometry in conjunction with airstream simulation used in diANA allows for millimeter accurate determination of its position, shape, and extent in all three dimensions. At the same time, it is possible to assess how airstream is influenced in context with the adjacent flow domain.

Factors such as size of the nose, body constitution, gender, ethnicity, and the respiratory minute volume affect the total nasal resistance but have little or no influence on the airstream pattern. This also applies to physiological phenomena such as nasal cycle, compensatory turbinate hyperplasia, or a moderate diffuse swelling of the entire inner lining. Airstream simulations performed by us and other research groups account for this assertion. ${ }^{13-22}$ Consequently, the results of diANA are relatively independent of the listed influences due to the topological comparison modus.

\section{Limitations}

Besides deformations of the nasal framework, the geometry of the flow domain can also be significantly modified through manifest diseases of the mucous membranes that is impractical or impossible to determine. Therefore, their presence should be excluded. However, in case of minor affections, for example, due to mucous formations, thin adhesions/synechiae, small polyps, or circumscribed perforations, reconstruction of a consistent geometry succeeds within certain limits through interpolation.

During strong inhalation, one can observe an inward movement of the alar wings due to the resulting transmural force. This is a phenomenon of fluid-structure interaction (FSI) and might serve as protection of the nasal or olfactory epithelium. ${ }^{17,35}$ The respective functional region in the nasal cavity works according to a Starling resistor limiting the inflow depending on its strength. ${ }^{36}$ Commonly, however, with rising physical stress a mouth bypass is initiated, and no onset of the so-called nasal valve mechanism occurs. Nevertheless, diANA intends to consider nasal breathing only at absolute rest enabling predominant or even exclusive respiration through the nose. Overall, effects of FSI appear to us to be negligible.

In case of incongruity of airflow perception and diANA results, determining to what extent problems with reception or the subsequent signal processing are involved, is challenging. Using diANA, one has to be aware that questions only regarding to the nose's morphology or the respective airflow can be addressed.

\section{Case}

A 28-year-old female patient, operated on the nasal framework elsewhere, complained about persisting impaired nasal breathing on the right side. A minor ipsilateral septum deviation was the only clinical correlate. The CT scan of the paranasal sinuses did not show a relevant chronic rhinosinusitis. Furthermore, rhinomanometry revealed resistance values on both sides in the normal range. There was uncertainty about the functional relevance of the septum deviation. Therefore, we applied diANA using the existing CT scan data. Qualitative comparison of the morphology and the pressure drop with the reference showed a substantial isthmus stenosis on the right side (-Fig. 4). In contrast to the measured resistances, the calculated values were in accordance with the patient's complains. We speculate that the rhinomanometer mask lateralized the cheek's soft tissue and, hence, generated false values. We proposed an extracorporeal septum correction, introduced by Gubisch and colleagues, to the patient. ${ }^{37,38}$

This example illustrates our opinion that conventional assessment of the isthmus nasi is not sufficiently reliable. 

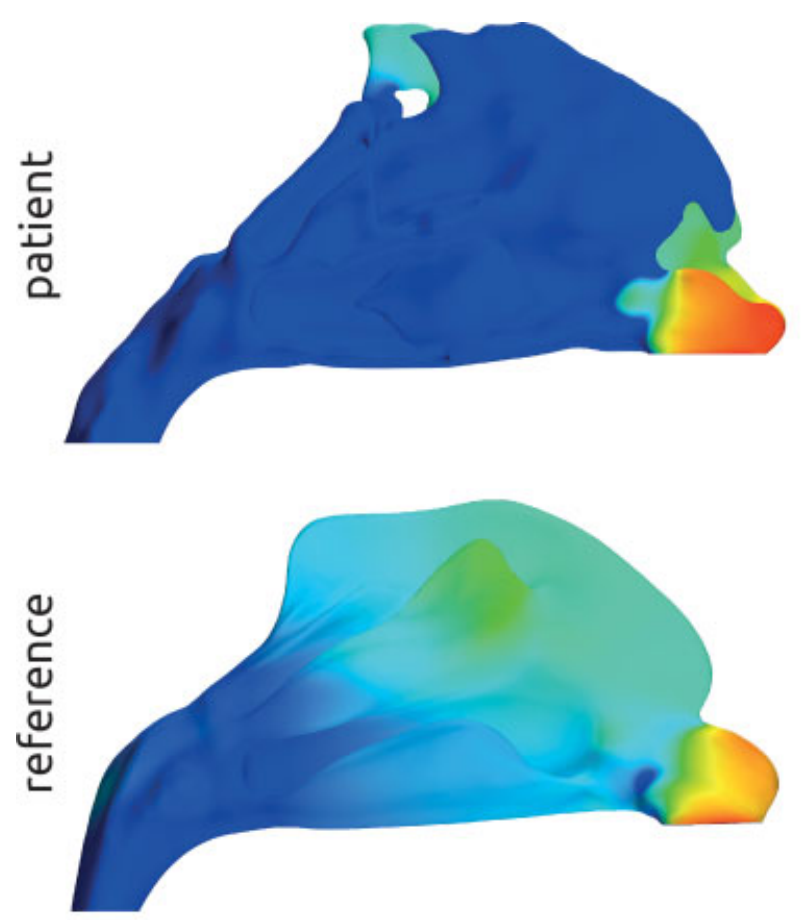

static pressure $(\mathrm{Pa})$

0

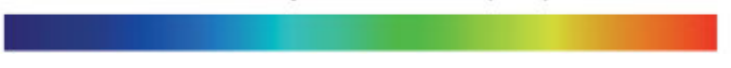

Fig. 4 Significant changes of the isthmus' morphology compared with the statistical shape model (SSM) reference on the right side, associated with increased pressure drop.

\section{Outlook}

Only recently we implemented diANA after 2 years of testing, but there is a constant need for enhancement. For example, we are continuously increasing the population of healthy nasal cavities to improve the quality and reliability of the SSM. Furthermore, complete automation of various processes, for example, segmentation, will help reduce the costs, diminish the investigator bias, and eventually support establishing the method.

Currently, we are working on the project making it possible to display obstructing regions or structures within the nasal cavity in terms of augmented reality during endoscopy or navigated surgery.

\section{Conclusion}

diANA is a new tool for the evaluation of the intranasal airstream. We have developed a technique that allows, in contrast to established methods, for a more comprehensive understanding of compromised nasal breathing and flowrelated changes of the inner milieu. Using diANA intends to distinguish between normal and abnormal nasal breathing as well as to detect the responsible findings in case of impairment. This has the potential to avoid unnecessary surgery on the one hand, and on the other to improve target-orientation and precision of functionally indicated operations on the nasal framework.

\section{Conflict of Interest}

Dr. Lamecker reports grants from Städtische Klinikum Karlsruhe and Charite - Universitätsmedizin Berlin, during the conduct of the study. The other authors have no conflict of interest.

\section{References}

1 Stewart M, Ferguson B, Fromer L. Epidemiology and burden of nasal congestion. Int J Gen Med 2010;3:37-45

2 Jessen M, Janzon L. Prevalence of non-allergic nasal complaints in an urban and a rural population in Sweden. Allergy 1989;44(08): 582-587

3 Hildebrandt T, Heppt WJ, Kertzscher U, Goubergrits L. The concept of rhinorespiratory homeostasis-a new approach to nasal breathing. Facial Plast Surg 2013;29(02):85-92

4 Vogt K, Jalowayski AA, Althaus W, et al. 4-Phase-Rhinomanometry (4PR)-basics and practice 2010. Rhinol Suppl 2010;21(21):1-50

5 Mlynski G, Beule A. Diagnostic methods of nasal respiratory function [in German]. HNO 2008;56(01):81-99

6 Mlynski G. Gestörte Funktion der oberen Atemwege wiederherstellende Verfahren bei gestörter Funktion der oberen Atemwege. Nasale Atmung. Laryngorhinootologie 2005;84:101-124

7 Digital Analysis of Nasal Breathing (diANA). Available at: https:// www.diana.doctor. Accessed November 5, 2018

8 Hildebrandt T, Osman J, Goubergrits L. Numerical flow simulation: a new method for assessing nasal breathing [in German]. HNO 2016;64(08):611-618

9 Osman J, Großmann F, Brosien K, Kertscher U, Goubergrits L, Hildebrandt T. Assessment of nasal resistance using computational fluid dynamics. Curr Direct Biomed Eng 2016;2(01):617-621

10 Quadrio M, Pipolo C, Corti S, et al. Review of computational fluid dynamics in the assessment of nasal air flow and analysis of its limitations. Eur Arch Otorhinolaryngol 2014;271(09):2349-2354

11 Goubergrits L, Schaller J, Kertzscher U, et al. Statistical wall shear stress maps of ruptured and unruptured middle cerebral artery aneurysms. J R Soc Interface 2012;9(69):677-688

12 Goubergrits L, Mevert R, Yevtushenko P, et al. The impact of MRIbased inflow for the hemodynamic evaluation of aortic coarctation. Ann Biomed Eng 2013;41(12):2575-2587

13 Brüning JJ, Goubergrits L, Heppt W, Zachow S, Hildebrandt T. Numerical analysis of nasal breathing: a pilot study. Facial Plast Surg 2017;33(04):388-395

14 Doorly DJ, Taylor DJ, Schroter RC. Mechanics of airflow in the human nasal airways. Respir Physiol Neurobiol 2008;163(13):100-110

15 Doorly DJ, Taylor DJ, Gambaruto AM, Schröter RC, Tolley N. Nasal architecture: form and flow. Philos Transact A Math Phys. Eng Sci 2008;366:3225-3246

16 Elad D, Naftali S, Rosenfeld M, Wolf M. Physical stresses at the airwall interface of the human nasal cavity during breathing. J Appl Physiol (1985) 2006;100(03):1003-1010

17 Hildebrandt T, Goubergrits L, Heppt WJ, Bessler S, Zachow S. Evaluation of the intranasal flow field through computational fluid dynamics. Facial Plast Surg 2013;29(02):93-98

18 Inthavong K, Shang Y, Tu J. Surface mapping for visualization of wall stresses during inhalation in a human nasal cavity. Respir Physiol Neurobiol 2014;190:54-61

19 Taylor DJ, Doorly DJ, Schroter RC. Inflow boundary profile prescription for numerical simulation of nasal airflow. J R Soc Interface 2010;7(44):515-527

20 Zachow S, Muigg P, Hildebrandt T, Doleisch H, Hege HC. Visual exploration of nasal airflow. IEEE Trans Vis Comput Graph 2009; 15(06):1407-1414

21 Zhao K, Jiang J. What is normal nasal airflow? A computational study of 22 healthy adults. Int Forum Allergy Rhinol 2014;4(06): 435-446 
22 Zhao K, Jiang J, Blacker K, et al. Regional peak mucosal cooling predicts the perception of nasal patency. Laryngoscope 2014;124 (03):589-595

23 Bruening J, Hildebrandt T, Bindernagel M, et al. A Statistical Shape Model of the Human Nasal Cavity. Paper presented at: 8th World Congress of Biomechanics; July, 2018; Dublin, Ireland

24 Lamecker $H$. Variational and statistical shape modeling for 3D geometry reconstruction [PhDThesis, in German]. Berlin, Germany: Freie Universität; 2009

25 Lamecker H,Zachow S. Statistical shape modeling of musculoskeletal structures and its applications. In: Zheng G, Li S, eds. Computational Radiology for Orthopedic Interventions. Lecture Notes in Computational Vision and Biomechanics. Cham: Springer; 2016: Vol. 23

26 Baumann C. Gene expression of endothelial cells under shear stress [Doctoral Thesis, in German]. Berlin, Germany: Freie Universität Berlin; 2002

27 Bongrazio M. Shear stress-dependent expression of proteins of the thrombospondin type 1 repeat (TSR) family in endothelial cells [Doctoral Thesis, in German]. Berlin, Germany: Freie Universität Berlin; 2004

28 Even-Tzur N, Kloog Y, Wolf M, Elad D. Mucus secretion and cytoskeletal modifications in cultured nasal epithelial cells exposed to wall shear stresses. Biophys J 2008;95(06):2998-3008

29 Schmidt VJ. Die Koordination des Gefäßverhaltens in der Mikrozirkulation wird durch Connexine mit spezifischen Eigenschaften vermittelt [Doctoral Thesis, in German]. Lübeck, Germany: Universität zu Lübeck; 2008

30 Davies PF. Flow-mediated endothelial mechanotransduction. Physiol Rev 1995;75(03):519-560

31 Hildebrandt T. Principles of modern septoplasty. In: Behrbohm H, Tardy EM, eds. Essentials of Septorhinoplasty. 2nd ed. New York, USA: Thieme; 2016:122-127

32 Wexler DB, Davidson TM. The nasal valve: a review of the anatomy, imaging, and physiology. Am J Rhinol 2004;18(03):143-150

33 Huizing EH, De Groot JAM. Functional Reconstructive Nasal Surgery. 1st ed. Stuttgart, Germany: Thieme; 2003

34 Proctor DF. The upper airway. In: Proctor DF, Anderson IB, eds. The Nose. Upper Airway Physiology and the Atmospheric Environment. New York, NY: Elsevier, Urban \& Fischer; 1982:23-43

35 Hildebrandt T. Das Konzept der Rhinorespiratorischen Homöostase -ein neuer theoretischer Ansatz für die Diskussion physiologischer und physikalischer Zusammenhänge bei der Nasenatmung [Doctoral Thesis, in German]. Freiburg im Breisgau, Germany: AlbertLudwigs-Universität; 2011

36 Bridger GP, Proctor DF. Maximum nasal inspiratory flow and nasal resistance. Ann Otol Rhinol Laryngol 1970;79(03):481-488

37 Gubisch W. Twenty-five years experience with extracorporeal septoplasty. Facial Plast Surg 2006;22(04):230-239

38 Heppt W, Gubisch W. Septal surgery in rhinoplasty. Facial Plast Surg 2011;27(02):167-178 